\title{
Interpolation theorem for the $p$-harmonic transform
}

\author{
by \\ Luigi D’Onofrio (Napoli) and TAdeusz Iwaniec (Syracuse, NY)
}

To Olek Petczyński on his 70th birthday

\begin{abstract}
We establish an interpolation theorem for a class of nonlinear operators in the Lebesgue spaces $\mathscr{L}^{s}\left(\mathbb{R}^{n}\right)$ arising naturally in the study of elliptic PDEs. The prototype of those PDEs is the second order $p$-harmonic equation $\operatorname{div}|\nabla u|^{p-2} \nabla u=\operatorname{div} \mathfrak{f}$. In this example the $p$-harmonic transform is essentially inverse to $\operatorname{div}\left(|\nabla|^{p-2} \nabla\right)$. To every vector field $\mathfrak{f} \in \mathscr{L}^{q}\left(\mathbb{R}^{n}, \mathbb{R}^{n}\right)$ our operator $\mathscr{H}_{p}$ assigns the gradient of the solution, $\mathscr{H}_{p} \mathfrak{f}=\nabla u \in$ $\mathscr{L}^{p}\left(\mathbb{R}^{n}, \mathbb{R}^{n}\right)$. The core of the matter is that we go beyond the natural domain of definition of this operator. Because of nonlinearity our arguments require substantial innovations as compared with the classical interpolation theory of Riesz, Thorin and Marcinkiewicz. The subject is largely motivated by recent developments in geometric function theory.
\end{abstract}

1. Introduction. The general theme running through this article is a type of nonlinear PDEs whose prototype is the well known $p$-harmonic equation in $\mathbb{R}^{n}$ :

$$
\operatorname{div}|\mathfrak{a}+\nabla u|^{p-2}(\mathfrak{a}+\nabla u)=\operatorname{div} \mathfrak{b}, \quad 1<p<\infty .
$$

Its natural setting is for the functions $u$ in the Sobolev class $\mathscr{W}^{1, p}\left(\mathbb{R}^{n}, \mathbb{R}\right)\left({ }^{1}\right)$, where $\mathfrak{a}=\left(a^{1}, \ldots, a^{n}\right) \in \mathscr{L}^{p}\left(\mathbb{R}^{n}, \mathbb{R}^{n}\right)$ and $\mathfrak{b}=\left(b^{1}, \ldots, b^{n}\right) \in \mathscr{L}^{q}\left(\mathbb{R}^{n}, \mathbb{R}^{n}\right)$ are given vector fields. Throughout this text, $\mathscr{L}^{p}$ and $\mathscr{L}^{q}$ will always be dual to each other. Precisely, the exponents $p$ and $q$ will satisfy Hölder's relation $p+q=p \cdot q<\infty$. The case $p=q=2$ gives rise to a linear equation and, therefore, the solution $\nabla u \in \mathscr{L}^{2}\left(\mathbb{R}^{n}, \mathbb{R}^{n}\right)$ is expressed by means of singular integrals,

$$
\nabla u=\mathscr{R}\langle\mathscr{R} \mid \mathfrak{a}-\mathfrak{b}\rangle=\mathscr{R} \sum\left(\mathscr{R}_{i} a^{i}-\mathscr{R}_{i} b^{i}\right)
$$

2000 Mathematics Subject Classification: 35J60, 41A05, 47B38.

Iwaniec was supported by NSF grant DMS-0301582. D'Onofrio was partially supported by GNAMPA. This research was done while D'Onofrio was visiting Mathematics Department at Syracuse University. He wishes to thank SU for support and hospitality.

$\left({ }^{1}\right)$ The notation $\mathscr{W}^{1, p}\left(\mathbb{R}^{n}, \mathbb{V}\right)$ stands for the Sobolev class of functions (modulo constants) defined in $\mathbb{R}^{n}$ and with values in a finite-dimensional inner product space $\mathbb{V}$, whose first order partials belong to $\mathscr{L}^{p}\left(\mathbb{R}^{n}, \mathbb{V}\right)$. These functions need not be $\mathscr{L}^{p}$-integrable, as $\mathbb{R}^{n}$ has infinite measure. 
where $\mathscr{R}=\left(\mathscr{R}_{1}, \ldots, \mathscr{R}_{n}\right)$ are the Riesz transforms in $\mathbb{R}^{n}$. In a similar fashion we define a nonlinear operator

$$
\mathscr{H}=\mathscr{H}_{p q}: \mathscr{L}^{p}\left(\mathbb{R}^{n}, \mathbb{R}^{n}\right) \times \mathscr{L}^{q}\left(\mathbb{R}^{n}, \mathbb{R}^{n}\right) \rightarrow \mathscr{L}^{p}\left(\mathbb{R}^{n}, \mathbb{R}^{n}\right) \times \mathscr{L}^{q}\left(\mathbb{R}^{n}, \mathbb{R}^{n}\right),
$$

which carries a given pair $(\mathfrak{a}, \mathfrak{b}) \in \mathscr{L}^{p}\left(\mathbb{R}^{n}, \mathbb{R}^{n}\right) \times \mathscr{L}^{q}\left(\mathbb{R}^{n}, \mathbb{R}^{n}\right)$ of vector fields to a curl-div couple that consists of the curl free gradient field $\nabla u$ and the divergence free vector field $|\mathfrak{a}+\nabla u|^{p-2}(\mathfrak{a}+\nabla u)-\mathfrak{b}$. This operator (p-harmonic transform for references) acts continuously from $\mathscr{L}^{p}\left(\mathbb{R}^{n}, \mathbb{R}^{n}\right) \times$ $\mathscr{L}^{q}\left(\mathbb{R}^{n}, \mathbb{R}^{n}\right)$ into itself.

There have been numerous variants of the $p$-harmonic equation, each of great interest in geometric function theory [BI.1], [IM], [Re], [Ri], [HKM], [MaZ]. By way of illustration, we recall the energy integral

$$
\mathscr{E}[f]=\int_{\Omega}|D f(x)|_{G(x)}^{n} \mathrm{~d} x
$$

for a quasiconformal deformation $f: \Omega \rightarrow \mathbb{R}^{n}$ of an open region $\Omega \subset \mathbb{R}^{n}$. The norm of the differential matrix $D f(x) \in \mathbb{R}^{n \times n}$ is computed with respect to a given Riemannian tensor on $\Omega$. This tensor is represented by a symmetric positive definite matrix function $G(x)=\left[G_{i j}(x)\right]$. In this setting the norm of a matrix $\xi \in \mathbb{R}^{n \times n}$ depends on the point $x \in \mathbb{R}^{n}$ and is given by

$$
|\xi|_{G(x)}=\sqrt{\langle\xi G(x) \mid \xi\rangle}
$$

Quasiconformal mappings, being minimizers of the energy integral, satisfy the corresponding Euler-Lagrange system of partial differential equations

$$
\operatorname{Div} \mathbf{A}(x, D f)=0 \quad \text { in } \Omega,
$$

where $\mathbf{A}(x):, \mathbb{R}^{n \times n} \rightarrow \mathbb{R}^{n \times n}$ is a nonlinear function of matrices. Explicitly, we have the formula

$$
\mathbf{A}(x, \xi)=\langle\xi G(x) \mid \xi\rangle^{(n-2) / 2} \xi G(x) \quad \text { for }(x, \xi) \in \Omega \times \mathbb{R}^{n \times n} .
$$

This system is understood in the sense of Schwartz distributions in which the divergence operator Div : $\mathscr{D}^{\prime}\left(\Omega, \mathbb{R}^{n \times n}\right) \rightarrow \mathscr{D}^{\prime}\left(\Omega, \mathbb{R}^{n}\right)$ carries matrix-valued distributions to vector-valued distributions. This operator is none other than the formal adjoint of the differential $D: \mathscr{D}^{\prime}\left(\Omega, \mathbb{R}^{n}\right) \rightarrow \mathscr{D}^{\prime}\left(\Omega, \mathbb{R}^{n \times n}\right)$. The reader may have noticed that the capital letter is being used to distinguish from the usual notation $\operatorname{div}: \mathscr{D}^{\prime}\left(\Omega, \mathbb{R}^{n}\right) \rightarrow \mathscr{D}^{\prime}(\Omega)$ for the divergence operator acting on vector fields.

Conversely, the $p$-harmonic functions (especially in two dimensions) are successfully treated by geometric methods of quasiconformal transformations (see for example [B] and [BI.2]).

Yet another example, also from geometric function theory [I.3], [IM], [ISS], is furnished by the equation

$$
\mathrm{d}^{*}|\mathfrak{a}+\mathrm{d} \alpha|^{p-2}(\mathfrak{a}+\mathrm{d} \alpha)=\mathrm{d}^{*} \mathfrak{b} .
$$


Here we look for a differential form $\alpha$ of degree $l-1$ in the Sobolev space $\mathscr{W}^{1, p}\left(\mathbb{R}^{n}, \bigwedge^{l-1}\right)$, where $\mathfrak{a} \in \mathscr{L}^{p}\left(\mathbb{R}^{n}, \bigwedge^{l}\right)$ and $\mathfrak{b} \in \mathscr{L}^{q}\left(\mathbb{R}^{n}, \bigwedge^{l+1}\right)$ are given differential forms. There is a very useful device that allows us to pass from a nonlinear $p$-harmonic equation to its Hodge dual $q$-harmonic equation [I.3]. This device has arisen from the concept of conjugate harmonic functions and the notion of stream functions [B]. Conjugate fields play an important role in the modern theory of quasiregular mappings [I.3], [IM]. Let us illustrate this idea in the case of the $p$-harmonic equation for differential forms. The Poincaré lemma ensures that there exists $\beta \in \mathscr{W}^{1, q}\left(\mathbb{R}, \bigwedge^{l+1}\right)$ such that

$$
|\mathfrak{a}+\mathrm{d} \alpha|^{p-2}(\mathfrak{a}+\mathrm{d} \alpha)=\mathfrak{b}+\mathrm{d}^{*} \beta .
$$

The term $\mathrm{d} \alpha$ can easily be eliminated by solving the equation for $\mathfrak{a}+\mathrm{d} \alpha$ and applying the exterior derivative. In this way, we arrive at the Hodge dual equation for $\mathrm{d}^{*} \beta$,

$$
\left|\mathfrak{b}+\mathrm{d}^{*} \beta\right|^{q-2}\left(\mathfrak{b}+\mathrm{d}^{*} \beta\right)=\mathfrak{a}+\mathrm{d} \alpha .
$$

Hence

$$
\mathrm{d}\left|\mathfrak{b}+\mathrm{d}^{*} \beta\right|^{q-2}\left(\mathfrak{b}+\mathrm{d}^{*} \beta\right)=\mathrm{d} \mathfrak{a} .
$$

The primary benefit from this transformation is that we need only consider the case $p \geqslant 2$. This of course requires knowing the $p$-harmonic theory with $p \geqslant 2$ in sufficient generality that includes differential forms. With this observation in mind it becomes natural to introduce the nonlinear operator $\mathscr{H}=\mathscr{H}_{p q}: \mathscr{L}^{p}\left(\mathbb{R}^{n}, \bigwedge^{l}\right) \times \mathscr{L}^{q}\left(\mathbb{R}^{n}, \bigwedge^{l}\right) \rightarrow \mathscr{L}^{p}\left(\mathbb{R}^{n}, \bigwedge^{l}\right) \times \mathscr{L}^{q}\left(\mathbb{R}^{n}, \bigwedge^{l}\right)$, defined by the rule $\mathscr{H}(\mathfrak{a}, \mathfrak{b})=\left(\mathrm{d} \alpha, \mathrm{d}^{*} \beta\right)$. However, for full generality, we shall consider two differential operators of the first order with constant coefficients,

$$
\left\{\begin{array}{l}
\mathscr{A}: \mathscr{D}^{\prime}\left(\mathbb{R}^{n}, \mathbb{X}\right) \rightarrow \mathscr{D}^{\prime}\left(\mathbb{R}^{n}, \mathbb{V}\right), \\
\mathscr{B}: \mathscr{D}^{\prime}\left(\mathbb{R}^{n}, \mathbb{Y}\right) \rightarrow \mathscr{D}^{\prime}\left(\mathbb{R}^{n}, \mathbb{V}\right),
\end{array}\right.
$$

where $\mathbb{X}, \mathbb{Y}$ and $\mathbb{V}$ are arbitrary finite-dimensional inner product spaces. These operators are to be supplemented with a suitable set of conditions, called ellipticity hypotheses. The references for these conditions are [GV], [IS.2]. Rather than discuss them in detail, we shall formulate the required properties of $\mathscr{A}$ and $\mathscr{B}$ and only illustrate the situation by examples. To this end, we consider $\mathscr{A}$ and $\mathscr{B}$ as elements of two short differential sequences:

$$
\left\{\begin{array}{l}
\mathscr{W}^{1, s}\left(\mathbb{R}^{n}, \mathbb{X}\right) \stackrel{\mathscr{A}}{\rightarrow} \mathscr{L}^{s}\left(\mathbb{R}^{n}, \mathbb{V}\right) \stackrel{\mathscr{A}^{*}}{\rightarrow} \mathscr{D}^{\prime}\left(\mathbb{R}^{n}, \mathbb{X}\right), \\
\mathscr{W}^{1, s}\left(\mathbb{R}^{n}, \mathbb{Y}\right) \stackrel{\mathscr{B}}{\rightarrow} \mathscr{L}^{s}\left(\mathbb{R}^{n}, \mathbb{V}\right) \stackrel{\mathscr{B}^{*}}{\rightarrow} \mathscr{D}^{\prime}\left(\mathbb{R}^{n}, \mathbb{Y}\right),
\end{array}\right.
$$

where $\mathscr{A}^{*}$ and $\mathscr{B}^{*}$ are formal adjoints to $\mathscr{A}$ and $\mathscr{B}$, respectively. These adjoints are defined by the usual rule of integration by parts

$$
\int_{\mathbb{R}^{n}}\langle\mathscr{A} \alpha \mid \phi\rangle=\int_{\mathbb{R}^{n}}\left\langle\alpha \mid \mathscr{A}^{*} \phi\right\rangle,
$$




$$
\int_{\mathbb{R}^{n}}\langle\mathscr{B} \beta \mid \phi\rangle=\int_{\mathbb{R}^{n}}\left\langle\beta \mid \mathscr{B}^{*} \phi\right\rangle
$$

for all $\alpha \in \mathscr{C}^{\infty}\left(\mathbb{R}^{n}, \mathbb{X}\right), \beta \in \mathscr{C}^{\infty}\left(\mathbb{R}^{n}, \mathbb{Y}\right)$ and $\phi \in \mathscr{C}_{0}^{\infty}\left(\mathbb{R}^{n}, \mathbb{V}\right)$. Here the angular brackets are used to denote the inner products in $\mathbb{X}, \mathbb{Y}$ and $\mathbb{V}$.

In relation to the differential sequences at (8), we now impose the following conditions on $\mathscr{A}$ and $\mathscr{B}$ :

$$
\left\{\begin{array}{l}
\operatorname{Ker} \mathscr{A}^{*}=\operatorname{Im} \mathscr{B} \\
\operatorname{Im} \mathscr{A}=\operatorname{Ker} \mathscr{B}^{*}
\end{array}\right.
$$

In other words the following sequences are exact for every $1<s<\infty$ :

$$
\left\{\begin{array}{l}
\mathscr{W}^{1, s}\left(\mathbb{R}^{n}, \mathbb{X}\right) \stackrel{\mathscr{A}}{\rightarrow} \mathscr{L}^{s}\left(\mathbb{R}^{n}, \mathbb{V}\right) \stackrel{\mathscr{B}^{*}}{\longrightarrow} \mathscr{D}^{\prime}\left(\mathbb{R}^{n}, \mathbb{Y}\right), \\
\mathscr{W}^{1, s}\left(\mathbb{R}^{n}, \mathbb{Y}\right) \stackrel{\mathscr{B}}{\rightarrow} \mathscr{L}^{s}\left(\mathbb{R}^{n}, \mathbb{V}\right) \stackrel{\mathscr{A}^{*}}{\rightarrow} \mathscr{D}^{\prime}\left(\mathbb{R}^{n}, \mathbb{X}\right)
\end{array}\right.
$$

See Section 2 for specific examples. We refer to these as elliptic complexes. In analogy with (3) and (6) we consider a pair of conjugate differential equations

$$
\left\{\begin{array}{l}
\mathscr{A}^{*} \mathbf{A}(x, \mathfrak{a}+\mathscr{A} \alpha)=\mathscr{A}^{*} \mathfrak{b} \\
\mathscr{B}^{*} \mathbf{B}(x, \mathfrak{b}+\mathscr{B} \beta)=\mathscr{B}^{*} \mathfrak{a}
\end{array}\right.
$$

where $\mathbf{A}, \mathbf{B}: \mathbb{R}^{n} \times \mathbb{V} \rightarrow \mathbb{V}$ are given nonlinear mappings. Our investigations require Carathéodory type hypotheses. The first two hypotheses are concerned with measurability and continuity of $\mathbf{A}(x, \xi)$ :

- $x \mapsto \mathbf{A}(x, \xi)$ is measurable in $x \in \Omega$ for every $\xi \in \mathbb{V}$.

- $\xi \mapsto \mathbf{A}(x, \xi)$ is continuous in $\mathbb{V}$ for almost every $x \in \Omega$.

These are important assumptions, for they guarantee that the composite function $\mathbf{A}(x, \xi(x))$ is measurable in $\Omega$, provided $\xi: \Omega \rightarrow \mathbb{V}$ is measurable (Scorza-Dragoni Theorem).

The second set of hypotheses is concerned with the ellipticity of the equation (13):

$$
\begin{aligned}
\mathbf{A}(x, 0) & \equiv 0 \\
\langle\mathbf{A}(x, \xi)-\mathbf{A}(x, \zeta) \mid \xi-\zeta\rangle & \geqslant K^{-1}|\xi-\zeta|^{2}(|\xi|+|\zeta|)^{p-2}, \\
|\mathbf{A}(x, \xi)-\mathbf{A}(x, \zeta)| \cdot|\xi-\zeta| & \leqslant K|\xi-\zeta|^{2}(|\xi|+|\zeta|)^{p-2}
\end{aligned}
$$

for all $\xi, \zeta \in \mathbb{V}$ and almost every $x \in \mathbb{R}^{n}$. Here $1<p<\infty$ is a given exponent. The number $K \geqslant 1$ will hereafter be referred to as the ellipticity constant. The inequality (14b) is often expressed by saying that $\mathbf{A}(x, \xi)$ is monotone in $\xi \in \mathbb{V}$. As for the mapping $\mathbf{B}(x):, \mathbb{V} \rightarrow \mathbb{V}$, the observant reader may have noticed that $\mathbf{A}(x):, \mathbb{V} \rightarrow \mathbb{V}$ is invertible. We then find $\mathbf{B}(x$,$) to be the inverse of \mathbf{A}(x$,$) , in symbols$

$$
\mathbf{A}(x,) \circ \mathbf{B}(x,)=\mathbf{B}(x,) \circ \mathbf{A}(x,)=\mathbf{I}: \mathbb{V} \rightarrow \mathbb{V} .
$$


This being so, conditions (14a)-(14c) entail similar ellipticity bounds for B:

$$
\begin{aligned}
\mathbf{B}(x, 0) & \equiv 0 \\
\langle\mathbf{B}(x, \xi)-\mathbf{B}(x, \zeta) \mid \xi-\zeta\rangle & \geqslant L^{-1}|\xi-\zeta|^{2}(|\xi|+|\zeta|)^{q-2} \\
|\mathbf{B}(x, \xi)-\mathbf{B}(x, \zeta)| \cdot|\xi-\zeta| & \leqslant L|\xi-\zeta|^{2}(|\xi|+|\zeta|)^{q-2}
\end{aligned}
$$

with an ellipticity constant $L \geqslant 1$. Conversely, these conditions imply (14a)(14c).

Given $\mathfrak{a} \in \mathscr{L}^{p}\left(\mathbb{R}^{n}, \mathbb{V}\right)$ and $\mathfrak{b} \in \mathscr{L}^{q}\left(\mathbb{R}^{n}, \mathbb{V}\right)$ the Minty-Browder theory of monotone operators [Mi], $[\mathrm{Br}]$ tells us that the solutions $\mathscr{A} \alpha \in \mathscr{L}^{p}\left(\mathbb{R}^{n}, \mathbb{V}\right)$ and $\mathscr{B} \beta \in \mathscr{L}^{q}\left(\mathbb{R}^{n}, \mathbb{V}\right)$ to the equations (13) exist and are uniquely determined by $\mathfrak{a}$ and $\mathfrak{b}$. This allows us to speak of the operator

$$
\mathscr{H}=\mathscr{H}_{\mathrm{AB}}: \mathscr{L}^{p}\left(\mathbb{R}^{n}, \mathbb{V}\right) \times \mathscr{L}^{q}\left(\mathbb{R}^{n}, \mathbb{V}\right) \rightarrow \mathscr{L}^{p}\left(\mathbb{R}^{n}, \mathbb{V}\right) \times \mathscr{L}^{q}\left(\mathbb{R}^{n}, \mathbb{V}\right)
$$

carrying the pair $(\mathfrak{a}, \mathfrak{b})$ to $(\mathscr{A} \alpha, \mathscr{B} \beta)$. Simple integration will reveal that

$$
\int_{\mathbb{R}^{n}}\left(|\mathscr{A} \alpha|^{p}+|\mathscr{B} \beta|^{q}\right) \preccurlyeq \int_{\mathbb{R}^{n}}\left(|\mathfrak{a}|^{p}+|\mathfrak{b}|^{q}\right) .
$$

Throughout this text whenever we write $\preccurlyeq$ we mean that the inequality holds with some positive constant, called implied constant. The reader will easily recognize from the context which parameters this constant might depend on. Let us emphasize that the implied constant may vary from line to line.

Recent major advances in geometric function theory depend upon estimates of $\mathscr{H}_{\mathrm{AB}}$ beyond the above domain of definition (see [IM]). The purpose of this paper is to establish interpolation properties of the operator $\mathscr{H}_{\mathrm{AB}}$. Before stating our conclusions, we need more notation. Throughout this text the Hölder conjugate exponents $1<p, q<\infty$ and the structural mappings $\mathbf{A}(x),, \mathbf{B}(x):, \mathbb{V} \rightarrow \mathbb{V}$ will be fixed. Given a pair of vectors $\mathbf{h}=(\mathbf{a}, \mathbf{b}) \in \mathbb{V} \times \mathbb{V}$, we define

$$
[\mathbf{h}]=|\mathbf{a}|^{p}+|\mathbf{b}|^{q} .
$$

Also, given a pair of functions $\mathfrak{h}=(\mathfrak{a}, \mathfrak{b}) \in \mathscr{L}^{p}\left(\mathbb{R}^{n}, \mathbb{V}\right) \times \mathscr{L}^{q}\left(\mathbb{R}^{n}, \mathbb{V}\right)$, we write

$$
\llbracket \mathfrak{h} \rrbracket=\int_{\mathbb{R}^{n}}\left(|\mathfrak{a}(x)|^{p}+|\mathfrak{b}(x)|^{q}\right) \mathrm{d} x .
$$

In this notation, the inequality (17) reads

$$
\llbracket \mathscr{H} \mathfrak{h} \rrbracket \preccurlyeq \llbracket \mathfrak{h} \rrbracket
$$

for every $\mathfrak{h}=(\mathfrak{a}, \mathfrak{b}) \in \mathscr{L}^{p}\left(\mathbb{R}^{n}, \mathbb{V}\right) \times \mathscr{L}^{q}\left(\mathbb{R}^{n}, \mathbb{V}\right)$. In fact, we even have an estimate that yields Hölder continuity of the operator $\mathscr{H}=\mathscr{H}_{\mathrm{AB}}$ :

$$
\llbracket \mathscr{H} \mathfrak{h}_{1}-\mathscr{H} \mathfrak{h}_{2} \rrbracket \preccurlyeq \llbracket \mathfrak{h}_{1}-\mathfrak{h}_{2} \rrbracket^{\theta}\left(\llbracket \mathfrak{h}_{1} \rrbracket+\llbracket \mathfrak{h}_{2} \rrbracket\right)^{1-\theta} .
$$

The largest possible exponent $\theta$ is $\min \{p-1, q-1\}$. 
Definition 1.1. The operator $\mathscr{H}_{\mathrm{AB}}$ is said to be of weak type $\lambda$, where $\lambda \geqslant \max \{1 / p, 1 / q\}$, if for every $t>0$,

$$
\operatorname{meas}\left\{x \in \mathbb{R}^{n} ;[\mathscr{H} \mathfrak{h}(x)]>t\right\} \preccurlyeq t^{-\lambda} \int_{\mathbb{R}^{n}}[\mathfrak{h}(x)]^{\lambda} \mathrm{d} x
$$

whenever $[\mathfrak{h}] \in \mathscr{L}^{\lambda}\left(\mathbb{R}^{n}, \mathbb{V}\right) \cap \mathscr{L}^{1}\left(\mathbb{R}^{n}, \mathbb{V}\right)$.

This is certainly the case if $\mathscr{H}$ is of strong type $\lambda$, meaning that

$$
\llbracket \mathscr{H} \mathfrak{h} \rrbracket_{\lambda}:=\left(\int_{\mathbb{R}^{n}}[\mathscr{H} \mathfrak{h}(x)]^{\lambda} \mathrm{d} x\right)^{1 / \lambda} \preccurlyeq\left(\int_{\mathbb{R}^{n}}[\mathfrak{h}(x)]^{\lambda} \mathrm{d} x\right)^{1 / \lambda}=\llbracket \mathfrak{h} \rrbracket_{\lambda} .
$$

Let us stress explicitly that the assumption $[\mathfrak{h}] \in \mathscr{L}^{1}\left(\mathbb{R}^{n}, \mathbb{V}\right)$ is necessary in order to speak of $\mathscr{H} \mathfrak{h}$. The question of whether $\mathscr{H}$ extends continuously to the entire space $\mathscr{L}^{\lambda}\left(\mathbb{R}^{n}, \mathbb{V}\right)$ remains open if $1>\lambda \geqslant \max \{1 / p, 1 / q\}$. The affirmative answer for $\lambda>1$ is given in [DI].

Theorem (Interpolation Theorem). If $\mathscr{H}_{\mathbf{A B}}$ is of weak type $\lambda$ for some $\lambda \geqslant \max (1 / p, 1 / q)$, then it is of strong type $\tau$ for every $\tau$ between 1 and $\lambda$, that is, $|1-\tau|<|1-\lambda|$.

Our arguments follow closely the well known proof of the Marcinkiewicz Interpolation Theorem for linear operators $[\mathrm{M}]$, [S]. However, we will touch upon some of the delicate but critical points due to nonlinearity of $\mathscr{H}_{\mathrm{AB}}$. There are several cases in which we are able to identify the parameters $\lambda$. First of all, $\mathscr{H}_{\mathrm{AB}}$ is always of strong type $\lambda$ if $\lambda$ is sufficiently close to 1 . This is essentially due to remarkable recent advances in the theory of various types of linear and nonlinear commutators [CCRSW], [RW], [IS.1], [I.4]. Estimates of $p$-harmonic type operators in the so-called grand $\mathscr{L}^{p}$-spaces can be found in [GIS]. They became particularly useful in the study of nonlinear PDEs with a measure on the right hand side. For a comprehensive treatment of estimates below the natural Sobolev exponent, that is, for $\lambda<1$, we refer the reader to [IS.1] and the bibliography given there. Estimates for large values of $\lambda$ lie deeply in the $\mathscr{C}^{1, \alpha}$-regularity theory of the $p$-harmonic equations [U], [Uh], effectively exploited in [I.1] and [I.2]. By way of digression, sharp regularity results for $p$-harmonic functions in the plane (and their surprising behavior as $p \rightarrow 1$ ) are available in [IMa]. Estimates for large exponents allow us to extend the $p$-harmonic operator to BMO-spaces (see $[\mathrm{DM}])$. Less related to our investigation is the study of the so-called infinite Laplacian and its viscosity solutions [JLM].

Let us look at particular situations of this kind in which $\mathfrak{a}=0$ or $\mathfrak{b}=0$. In the first case we are reduced to the equation

$$
\mathscr{A}^{*} \mathbf{A}(x, \mathscr{A} \alpha)=\mathscr{A}^{*} \mathfrak{b}, \quad \mathfrak{b} \in \mathscr{L}^{q}\left(\mathbb{R}^{n}, \mathbb{V}\right)
$$

which defines an operator $\mathscr{H}_{\mathbf{A}}: \mathscr{L}^{q}\left(\mathbb{R}^{n}, \mathbb{V}\right) \rightarrow \mathscr{L}^{q}\left(\mathbb{R}^{n}, \mathbb{V}\right)$ by the rule $\mathscr{H}_{\mathbf{A}}(\mathfrak{b})=\mathbf{A}(x, \mathscr{A} \alpha)-\mathfrak{b} \in \mathscr{L}^{q}\left(\mathbb{R}^{n}, \mathbb{V}\right)$. Thus, $\mathscr{H}_{\mathbf{A}}(\mathfrak{b})=\mathscr{H}_{\mathbf{A B}}(0, \mathfrak{b})$. 
In the second case we are dealing with the Hodge dual B-harmonic equation

$$
\mathscr{B}^{*} \mathbf{B}(x, \mathscr{B} \beta)=\mathscr{B}^{*} \mathfrak{a}, \quad \mathfrak{a} \in \mathscr{L}^{p}\left(\mathbb{R}^{n}, \mathbb{V}\right),
$$

which defines an operator $\mathscr{H}_{\mathrm{B}}: \mathscr{L}^{p}\left(\mathbb{R}^{n}, \mathbb{V}\right) \rightarrow \mathscr{L}^{p}\left(\mathbb{R}^{n}, \mathbb{V}\right)$ by the rule $\mathscr{H}_{\mathbf{B}}(\mathfrak{a})=\mathbf{B}(x, \mathscr{B} \beta)-\mathfrak{a} \in \mathscr{L}^{p}\left(\mathbb{R}^{n}, \mathbb{V}\right)$. Thus, $\mathscr{H}_{\mathbf{B}}(\mathfrak{a})=\mathscr{H}_{\mathbf{A B}}(\mathfrak{a}, 0)$. As a corollary from our Theorem we see that

$$
\begin{aligned}
\left\|\mathscr{H}_{\mathbf{A}}(\mathfrak{b})\right\|_{\tau q} & \preccurlyeq\|\mathfrak{b}\|_{\tau q}, \\
\left\|\mathscr{H}_{\mathbf{B}}(\mathfrak{a})\right\|_{\tau p} & \preccurlyeq\|\mathfrak{a}\|_{\tau p},
\end{aligned}
$$

whenever $\mathfrak{a} \in \mathscr{L}^{p}\left(\mathbb{R}^{n}, \mathbb{V}\right) \cap \mathscr{L}^{\tau p}\left(\mathbb{R}^{n}, \mathbb{V}\right)$ and $\mathfrak{b} \in \mathscr{L}^{q}\left(\mathbb{R}^{n}, \mathbb{V}\right) \cap \mathscr{L}^{\tau q}\left(\mathbb{R}^{n}, \mathbb{V}\right)$. As a matter of fact our interest in the most general setting is not only theoretical and aesthetic: the generality is also necessary to make the proofs easier and transparent. The $p$-harmonic theory has a long history and many results, too numerous to discuss them all. We hope that the reader will find our references helpful.

2. Remarks on elliptic complexes. We find it useful and interesting to briefly review the concept of ellipticity in relation to the first order partial differential operators $\mathscr{A}$ and $\mathscr{B}$. Let us begin with the explicit formulas

$$
\mathscr{A}=\sum_{i=1}^{n} \mathfrak{A}_{i} \frac{\partial}{\partial x_{i}}, \quad \mathscr{B}=\sum_{i=1}^{n} \mathfrak{B}_{i} \frac{\partial}{\partial x_{i}},
$$

where the coefficients $\mathfrak{A}_{i} \in \operatorname{Hom}(\mathbb{X}, \mathbb{V})$ and $\mathfrak{B}_{i} \in \operatorname{Hom}(\mathbb{Y}, \mathbb{V})$ are given linear transformations. Then the coefficients of the formal adjoints

$$
\mathscr{A}^{*}=\sum_{i=1}^{n} \mathfrak{A}_{i}^{*} \frac{\partial}{\partial x_{i}}, \quad \mathscr{B}^{*}=\sum_{i=1}^{n} \mathfrak{B}_{i}^{*} \frac{\partial}{\partial x_{i}}
$$

are the transpose transformations $\mathfrak{A}_{i}^{*} \in \operatorname{Hom}(\mathbb{V}, \mathbb{X})$ and $\mathfrak{B}_{i}^{*} \in \operatorname{Hom}(\mathbb{V}, \mathbb{Y})$. The simplest example is furnished by the divergence operator $\mathscr{A}=\operatorname{div}=$ $-\langle\nabla, \cdot\rangle$ and its formal adjoint $\mathscr{A}^{*}=-\nabla=-\left(\frac{\partial}{\partial x_{1}}, \ldots, \frac{\partial}{\partial x_{n}}\right)$,

$$
\mathscr{W}^{1, s}\left(\mathbb{R}^{n}, \mathbb{R}^{n}\right) \stackrel{\operatorname{div}}{\longrightarrow} \mathscr{L}^{s}\left(\mathbb{R}^{n}, \mathbb{R}\right) \stackrel{-\nabla}{\longrightarrow} \mathscr{D}^{\prime}\left(\mathbb{R}^{n}, \mathbb{R}^{n}\right) .
$$

As for the operator $\mathscr{B}$, we associate with (27) the trivial differential sequence

$$
\mathscr{W}^{1, s}\left(\mathbb{R}^{n}, \mathbb{Y}\right) \stackrel{\mathscr{B}=0}{\longrightarrow} \mathscr{L}^{s}\left(\mathbb{R}^{n}, \mathbb{R}\right) \stackrel{\mathscr{B}^{*}=0}{\longrightarrow} \mathscr{D}^{\prime}\left(\mathbb{R}^{n}, \mathbb{Y}\right)
$$

Clearly, we have

$$
\operatorname{Ker} \nabla=0=\operatorname{Im} \mathscr{B}, \quad \operatorname{Im} \operatorname{div}=\mathscr{L}^{s}\left(\mathbb{R}^{n}, \mathbb{R}\right)=\operatorname{Ker} \mathscr{B}^{*} .
$$

This example leads to the equation $\nabla \mathbf{A}(x, \mathfrak{a}+\operatorname{div} F)=\nabla \mathfrak{b}$, rather uninteresting. Nevertheless, an interesting $p$-harmonic type equation arises when 
we switch the divergence with the gradient, namely

$$
\mathscr{W}^{1, s}\left(\mathbb{R}^{n}, \mathbb{R}\right) \stackrel{\nabla}{\rightarrow} \mathscr{L}^{s}\left(\mathbb{R}^{n}, \mathbb{R}^{n}\right) \stackrel{-\operatorname{div}}{\longrightarrow} \mathscr{D}^{\prime}\left(\mathbb{R}^{n}, \mathbb{R}\right) .
$$

We must attach to this sequence another one that ensures conditions (11). With a little effort we find that the following differential sequence works well for our purpose:

$$
\mathscr{W}^{1, s}\left(\mathbb{R}^{n}, \mathbb{R}_{\text {skew }}^{n \times n}\right) \stackrel{\text { Div }}{\longrightarrow} \mathscr{L}^{s}\left(\mathbb{R}^{n}, \mathbb{R}^{n}\right) \stackrel{\text { Curl }}{\longrightarrow} \mathscr{D}^{\prime}\left(\mathbb{R}^{n}, \mathbb{R}_{\text {skew }}^{n \times n}\right) .
$$

Here, the rotation operator Curl carries a vector field $\mathbf{F}=\left(F^{1}, \ldots, F^{n}\right)$ to the matrix function

$$
\operatorname{Curl} \mathbf{F}=\frac{1}{2}\left[\frac{\partial F^{i}}{\partial x_{j}}-\frac{\partial F^{j}}{\partial x_{i}}\right]_{i, j=1, \ldots, n} \in \mathbb{R}_{\mathrm{skew}}^{n \times n} .
$$

Thus Curl $\mathbf{F}$ is a distribution with values in the space of skew symmetric matrices, denoted by $\mathbb{R}_{\text {skew }}^{n \times n}$. Its formal adjoint is defined on skew symmetric matrix functions $\mathbf{M}=\left[M_{j}^{i}\right]$ by the rule

$$
\operatorname{Div} \mathbf{M}=\left[\sum_{\nu=1}^{n} \frac{\partial}{\partial x_{\nu}} \mathbf{M}_{i}^{\nu}\right]_{i=1, \ldots, n} .
$$

In other words, the $i$ th component of the vector field Div $\mathbf{M}$ is the divergence of the $i$ th column of $\mathbf{M}$. Clearly, we have $\operatorname{Ker}[\operatorname{div}]=\operatorname{Im}[\operatorname{Div}]$ and $\operatorname{Im}[\nabla]=$ Ker[Curl], as required. There are many examples of elliptic complexes, for which the reader may wish to consult [Uh], [GV] and [IS.2].

Associated with $\mathscr{A}$ and $\mathscr{B}$ is the elliptic Laplace-Beltrami operator

$$
-\triangle=\mathscr{A} \mathscr{A}^{*}+\mathscr{B} \mathscr{B}^{*}: \mathscr{W}^{2, s}\left(\mathbb{R}^{n}, \mathbb{V}\right) \rightarrow \mathscr{L}^{s}\left(\mathbb{R}^{n}, \mathbb{V}\right), \quad 1<s<\infty .
$$

This second order operator is the key instrument in the regularity of the operators $\mathscr{A}$ and $\mathscr{B}$. In much the same way as in [GV, Lemma 4$]$ we infer, by an approximation argument, the following

Lemma 2.1. The two spaces $\operatorname{Im} \mathscr{A} \subset \mathscr{L}^{p}\left(\mathbb{R}^{n}, \mathbb{V}\right)$ and $\operatorname{Im} \mathscr{B} \subset \mathscr{L}^{q}\left(\mathbb{R}^{n}, \mathbb{V}\right)$ are orthogonal, meaning that

$$
\int_{\mathbb{R}^{n}}\langle\mathscr{A} \alpha \mid \mathscr{B} \beta\rangle=0
$$

whenever $\alpha \in \mathscr{W}^{1, p}\left(\mathbb{R}^{n}, \mathbb{X}\right)$ and $\beta \in \mathscr{W}^{1, q}\left(\mathbb{R}^{n}, \mathbb{Y}\right)$.

We have already mentioned that the existence and uniqueness of the solutions of the system (13) follows by the Minty-Browder theory of monotone operators.

3. The natural domain. In this section we make preliminary computations for the equation

$$
\mathbf{A}(x, \mathfrak{a}+\mathscr{A} \alpha)=\mathfrak{b}+\mathscr{B} \beta
$$


and its dual

$$
\mathbf{B}(x, \mathfrak{b}+\mathscr{B} \beta)=\mathfrak{a}+\mathscr{A} \alpha .
$$

We shall consider these equations in the natural setting, meaning that $\mathfrak{a} \in \mathscr{L}^{p}\left(\mathbb{R}^{n}, \mathbb{V}\right)$ and $\alpha \in \mathscr{W}^{1, p}\left(\mathbb{R}^{n}, \mathbb{X}\right)$, whereas $\mathfrak{b} \in \mathscr{L}^{q}\left(\mathbb{R}^{n}, \mathbb{V}\right)$ and $\beta \in$ $\mathscr{W}^{1, q}\left(\mathbb{R}^{n}, \mathbb{Y}\right)$.

The easy strong type 1 estimate will get us started.

3.1. $\mathscr{H}$ is of strong type 1 . We aim to show that $\llbracket \mathscr{H} \mathfrak{h} \rrbracket \preccurlyeq \llbracket \mathfrak{h} \rrbracket$, which translates into (17). To this end, we integrate the inner product of $\mathfrak{a}+\mathscr{A} \alpha$ and the terms in $(29)$ :

$$
\int\langle\mathbf{A}(x, \mathfrak{a}+\mathscr{A} \alpha) \mid \mathfrak{a}+\mathscr{A} \alpha\rangle=\int\langle\mathfrak{b} \mid \mathfrak{a}+\mathscr{A} \alpha\rangle
$$

because $\mathscr{A} \alpha$ is orthogonal to $\mathscr{B} \beta$. The left hand side will be treated by using monotonicity of the mapping $\mathbf{A}(x$,$) . Precisely, we have$

$$
|\xi|^{p} \leqslant K\langle\mathbf{A}(x, \xi) \mid \xi\rangle,
$$

which is immediate from (14a) and (14b). Applying Hölder's inequality to the right hand side of (31) yields

$$
\|\mathfrak{a}+\mathscr{A} \alpha\|_{p}^{p} \leqslant K\|\mathscr{B} \beta\|_{q}\|\mathfrak{a}\|_{p}+K\|\mathfrak{b}\|_{q}\|\mathfrak{a}+\mathscr{A} \alpha\|_{p} .
$$

The term $\|\mathfrak{a}+\mathscr{A} \alpha\|_{p}$ will be absorbed by the left hand side once we separate it from $K\|\mathfrak{b}\|_{q}$ by using Young's inequality, that is, $x y \leqslant x^{q} / q+y^{p} / p$. It results in the inequality

$$
\|\mathfrak{a}+\mathscr{A} \alpha\|_{p}^{p} \leqslant K^{q}\|\mathfrak{b}\|_{q}^{q}+q K\|\mathscr{B} \beta\|_{q}\|\mathfrak{a}\|_{p} .
$$

From now on we adopt the notation $\preccurlyeq$ to avoid writing insignificant constants. Accordingly, we write

$$
\|\mathscr{A} \alpha\|_{p}^{p} \preccurlyeq\|\mathfrak{a}\|_{p}^{p}+\|\mathfrak{b}\|_{q}^{q}+\|\mathfrak{a}\|_{p}\|\mathscr{B} \beta\|_{q} .
$$

In much the same way, or by dualities, we obtain the analogous estimate for $\mathscr{B} \beta$ :

$$
\|\mathscr{B} \beta\|_{q}^{q} \preccurlyeq\|\mathfrak{a}\|_{p}^{p}+\|\mathfrak{b}\|_{q}^{q}+\|\mathfrak{b}\|_{q}\|\mathscr{A} \alpha\|_{p} .
$$

Finally, we add them up and use Young's inequality again so that the terms $\|\mathscr{B} \beta\|_{q}$ and $\|\mathscr{A} \alpha\|_{p}$ will be absorbed by the left hand side. This gives the desired estimate

$$
\|\mathscr{A} \alpha\|_{p}^{p}+\|\mathscr{B} \beta\|_{q}^{q} \preccurlyeq\|\mathfrak{a}\|_{p}^{p}+\|\mathfrak{b}\|_{q}^{q}
$$

which is a more explicit form of (19).

3.2. Hölder's exponent of $\mathscr{H}: \mathscr{L}^{p} \times \mathscr{L}^{q} \rightarrow \mathscr{L}^{p} \times \mathscr{L}^{q}$. Here we prove inequality (20). At the end we also show that the exponent $\theta=\min \{p-1$, $q-1\}$ is the best possible. Let $\mathfrak{h}_{1}=\left(\mathfrak{a}_{1}, \mathfrak{b}_{1}\right)$ and $\mathfrak{h}_{2}=\left(\mathfrak{a}_{2}, \mathfrak{b}_{2}\right)$. By the definition of $\mathscr{H}$, we have

$$
\mathscr{H} \mathfrak{h}_{1}=\left(\mathscr{A} \alpha_{1}, \mathscr{B} \beta_{1}\right), \quad \mathscr{H} \mathfrak{h}_{2}=\left(\mathscr{A} \alpha_{2}, \mathscr{B} \beta_{2}\right),
$$


where the first components $\mathscr{A} \alpha_{1}$ and $\mathscr{A} \alpha_{2}$ lie in $\mathscr{L}^{p}\left(\mathbb{R}^{n}, \mathbb{V}\right)$, while the second components $\mathscr{B} \beta_{1}$ and $\mathscr{B} \beta_{2}$ lie in $\mathscr{L}^{q}\left(\mathbb{R}^{n}, \mathbb{V}\right)$. These components are coupled in the equations

$$
\begin{array}{ll}
\mathbf{A}\left(x, \mathfrak{a}_{1}+\mathscr{A} \alpha_{1}\right)=\mathfrak{b}_{1}+\mathscr{B} \beta_{1}, & \mathbf{B}\left(x, \mathfrak{b}_{1}+\mathscr{B} \beta_{1}\right)=\mathfrak{a}_{1}+\mathscr{A} \alpha_{1}, \\
\mathbf{A}\left(x, \mathfrak{a}_{2}+\mathscr{A} \alpha_{2}\right)=\mathfrak{b}_{2}+\mathscr{B} \beta_{2}, & \mathbf{B}\left(x, \mathfrak{b}_{2}+\mathscr{B} \beta_{2}\right)=\mathfrak{a}_{2}+\mathscr{A} \alpha_{2},
\end{array}
$$

which we abbreviate to

$$
\begin{array}{ll}
\mathcal{A}_{1}=\mathfrak{a}_{1}+\mathscr{A} \alpha_{1}, & \mathcal{A}_{2}=\mathfrak{a}_{2}+\mathscr{A} \alpha_{2}, \\
\mathcal{B}_{1}=\mathfrak{b}_{1}+\mathscr{B} \beta_{1}, & \mathcal{B}_{2}=\mathfrak{b}_{2}+\mathscr{B} \beta_{2} .
\end{array}
$$

A simple application of the triangle inequality shows that $\left\|\mathcal{A}_{1}\right\|_{p} \preccurlyeq\left\|\mathfrak{a}_{1}\right\|_{p}+$ $\left\|\mathscr{A} \alpha_{1}\right\|_{p}$ and $\left\|\mathcal{B}_{1}\right\|_{q} \preccurlyeq\left\|\mathfrak{b}_{1}\right\|_{q}+\left\|\mathscr{B} \alpha_{1}\right\|_{q}$. By the strong type 1 estimate at (19), we have

$$
\begin{aligned}
& \left\|\mathcal{A}_{1}\right\|_{p}^{p}+\left\|\mathcal{B}_{1}\right\|_{q}^{q} \preccurlyeq \llbracket \mathfrak{h}_{1} \rrbracket, \\
& \left\|\mathcal{A}_{2}\right\|_{p}^{p}+\left\|\mathcal{B}_{2}\right\|_{q}^{q} \preccurlyeq \llbracket \mathfrak{h}_{2} \rrbracket .
\end{aligned}
$$

Returning to (33) and (34), we now subtract the equations and their dual counterparts to obtain

$$
\left\{\begin{array}{l}
\mathbf{A}\left(x, \mathcal{A}_{1}\right)-\mathbf{A}\left(x, \mathcal{A}_{2}\right)=\mathcal{B}_{1}-\mathcal{B}_{2}, \\
\mathcal{A}_{1}-\mathcal{A}_{2}=\mathbf{B}\left(x, \mathcal{A}_{1}\right)-\mathbf{B}\left(x, \mathcal{A}_{2}\right) .
\end{array}\right.
$$

Next we compute the inner products:

$$
\begin{aligned}
\left\langle\mathbf{A}\left(x, \mathcal{A}_{1}\right)-\mathbf{A}\left(x, \mathcal{A}_{2}\right) \mid \mathcal{A}_{1}-\mathcal{A}_{2}\right\rangle & =\left\langle\mathbf{B}\left(x, \mathcal{B}_{1}\right)-\mathbf{B}\left(x, \mathcal{B}_{2}\right) \mid \mathcal{B}_{1}-\mathcal{B}_{2}\right\rangle \\
& =\left\langle\mathcal{A}_{1}-\mathcal{A}_{2} \mid \mathcal{B}_{1}-\mathcal{B}_{2}\right\rangle \geqslant 0 .
\end{aligned}
$$

Nonnegativity of these terms, which is immediate from the monotonicity of $\mathbf{A}$ and $\mathbf{B}$, will be tacitly used throughout this text. The following three terms are essentially the same:

$$
\begin{aligned}
\left|\mathcal{A}_{1}-\mathcal{A}_{2}\right|^{2}\left(\left|\mathcal{A}_{1}\right|+\left|\mathcal{A}_{2}\right|\right)^{p-2} & \preccurlyeq \succcurlyeq\left\langle\mathcal{A}_{1}-\mathcal{A}_{2} \mid \mathcal{B}_{1}-\mathcal{B}_{2}\right\rangle \\
& \preccurlyeq \succcurlyeq\left|\mathcal{B}_{1}-\mathcal{B}_{2}\right|^{2}\left(\left|\mathcal{B}_{1}\right|+\left|\mathcal{B}_{2}\right|\right)^{q-2} .
\end{aligned}
$$

Of course the notation $X \preccurlyeq \succcurlyeq Y$ stands for the two inequalities $X \preccurlyeq Y$ and $Y \preccurlyeq X$.

Since the situation is symmetric with respect to $\mathbf{A}$ and $\mathbf{B}$, it involves no loss of generality to assume that $p \geqslant 2$. It is at this moment that we are going to brake the symmetry. From now on we take $1<q \leqslant 2 \leqslant p<\infty$. Hence we have

$$
\begin{aligned}
\left|\mathcal{A}_{1}-\mathcal{A}_{2}\right|^{p} & \leqslant\left|\mathcal{A}_{1}-\mathcal{A}_{2}\right|^{2}\left(\left|\mathcal{A}_{1}\right|+\left|\mathcal{A}_{2}\right|\right)^{p-2} \\
& \preccurlyeq \succcurlyeq\left\langle\mathcal{A}_{1}-\mathcal{A}_{2} \mid \mathcal{B}_{1}-\mathcal{B}_{2}\right\rangle \\
& \preccurlyeq \succcurlyeq\left|\mathcal{B}_{1}-\mathcal{B}_{2}\right|^{2}\left(\left|\mathcal{B}_{1}\right|+\left|\mathcal{B}_{2}\right|\right)^{q-2} \leqslant\left|\mathcal{B}_{1}-\mathcal{B}_{2}\right|^{q} .
\end{aligned}
$$


Then we integrate to obtain

$$
\begin{aligned}
\int_{\mathbb{R}^{n}} \mid \mathcal{A}_{1} & -\left.\mathcal{A}_{2}\right|^{p}+\int_{\mathbb{R}^{n}}\left|\mathcal{B}_{1}-\mathcal{B}_{2}\right|^{q} \leqslant 2 \int_{\mathbb{R}^{n}}\left|\mathcal{B}_{1}-\mathcal{B}_{2}\right|^{q} \\
& =2 \int_{\mathbb{R}^{n}}\left|\mathcal{B}_{1}-\mathcal{B}_{2}\right|^{q}\left(\left|\mathcal{B}_{1}\right|+\left|\mathcal{B}_{2}\right|\right)^{(q-2) q / 2}\left(\left|\mathcal{B}_{1}\right|+\left|\mathcal{B}_{2}\right|\right)^{(2-q) q / 2} \\
& \leqslant\left(\int_{\mathbb{R}^{n}}\left|\mathcal{B}_{1}-\mathcal{B}_{2}\right|^{2}\left(\left|\mathcal{B}_{1}\right|+\left|\mathcal{B}_{2}\right|\right)^{q-2}\right)^{q / 2}\left(\int_{\mathbb{R}^{n}}\left(\left|\mathcal{B}_{1}\right|+\left|\mathcal{B}_{2}\right|\right)^{q}\right)^{(2-q) / 2} \\
& \leqslant 2\left[\int_{\mathbb{R}^{n}}\left|\mathcal{B}_{1}-\mathcal{B}_{2}\right|^{2}\left(\left|\mathcal{B}_{1}\right|+\left|\mathcal{B}_{2}\right|\right)^{q-2}\right]^{q / 2}\left(\llbracket \mathfrak{h}_{1} \rrbracket+\llbracket \mathfrak{h}_{2} \rrbracket\right)^{(2-q) / 2} .
\end{aligned}
$$

The last step follows by (37) and (38). As we have already observed, the integral in brackets can be estimated as

$$
\begin{aligned}
\int_{\mathbb{R}^{n}}\left|\mathcal{B}_{1}-\mathcal{B}_{2}\right|^{2}\left(\left|\mathcal{B}_{1}\right|+\left|\mathcal{B}_{2}\right|\right)^{q-2} \\
\preccurlyeq \int_{\mathbb{R}^{n}}\left\langle\mathcal{A}_{1}-\mathcal{A}_{2} \mid \mathcal{B}_{1}-\mathcal{B}_{2}\right\rangle \\
=\int_{\mathbb{R}^{n}}\left\langle\mathfrak{a}_{1}-\mathfrak{a}_{2} \mid \mathfrak{b}_{1}-\mathfrak{b}_{2}\right\rangle+\int_{\mathbb{R}^{n}}\left\langle\mathfrak{a}_{1}-\mathfrak{a}_{2} \mid \mathscr{B} \beta_{1}-\mathscr{B} \beta_{2}\right\rangle \\
\quad+\int_{\mathbb{R}^{n}}\left\langle\mathscr{A} \alpha_{1}-\mathscr{A} \alpha_{2} \mid \mathfrak{b}_{1}-\mathfrak{b}_{2}\right\rangle+\int_{\mathbb{R}^{n}}\left\langle\mathscr{A} \alpha_{1}-\mathscr{A} \alpha_{2} \mid \mathscr{B} \beta_{1}-\mathscr{B} \beta_{2}\right\rangle .
\end{aligned}
$$

At this point we shall make use of the orthogonality of the subspaces $\operatorname{Im} \mathscr{A} \subset \mathscr{L}^{p}\left(\mathbb{R}^{n}, \mathbb{V}\right)$ and $\operatorname{Im} \mathscr{B} \subset \mathscr{L}^{q}\left(\mathbb{R}^{n}, \mathbb{V}\right)$, as discussed in Lemma 2.1. Accordingly, the last integral vanishes. The other integrals are treated by Hölder's inequality and we obtain

$$
\begin{aligned}
\int_{\mathbb{R}^{n}}\left|\mathcal{B}_{1}-\mathcal{B}_{2}\right|^{2}\left(\left|\mathcal{B}_{1}\right|\right. & \left.+\left|\mathcal{B}_{2}\right|\right)^{q-2} \\
\preccurlyeq & \left\|\mathfrak{a}_{1}-\mathfrak{a}_{2}\right\|_{p}\left\|\mathfrak{b}_{1}-\mathfrak{b}_{2}\right\|_{q}+\left\|\mathfrak{a}_{1}-\mathfrak{a}_{2}\right\|_{p}\left\|\mathscr{B} \beta_{1}-\mathscr{B} \beta_{2}\right\|_{q} \\
& \quad+\left\|\mathscr{A} \alpha_{1}-\mathscr{A} \alpha_{2}\right\|_{p}\left\|\mathfrak{b}_{1}-\mathfrak{b}_{2}\right\|_{q} \\
\leqslant & \llbracket \mathfrak{h}_{1}-\mathfrak{h}_{2} \rrbracket^{1 / p} \llbracket \mathfrak{h}_{1}-\mathfrak{h}_{2} \rrbracket^{1 / q}+\llbracket \mathfrak{h}_{1}-\mathfrak{h}_{2} \rrbracket^{1 / p} \llbracket \mathscr{H} \mathfrak{h}_{1}-\mathscr{H} \mathfrak{h}_{2} \rrbracket^{1 / q} \\
& +\llbracket \mathfrak{h}_{1}-\mathfrak{h}_{2} \rrbracket^{1 / q} \llbracket \mathscr{H} \mathfrak{h}_{1}-\mathscr{H} \mathfrak{h}_{2} \rrbracket^{1 / p}
\end{aligned}
$$

With this estimate the inequality (42) becomes

$$
\begin{aligned}
\int_{\mathbb{R}^{n}}\left|\mathcal{A}_{1}-\mathcal{A}_{2}\right|^{p}+\int_{\mathbb{R}^{n}} \mid \mathcal{B}_{1}- & \left.\mathcal{B}_{2}\right|^{q} \preccurlyeq\left(\llbracket \mathfrak{h}_{1} \rrbracket+\llbracket \mathfrak{h}_{2} \rrbracket\right)^{(2-q) / 2} \\
& \times\left(\llbracket \mathfrak{h}_{1}-\mathfrak{h}_{2} \rrbracket^{q / 2}+\llbracket \mathfrak{h}_{1}-\mathfrak{h}_{2} \rrbracket^{(q-1) / 2} \llbracket \mathscr{H} \mathfrak{h}_{1}-\mathscr{H} \mathfrak{h}_{2} \rrbracket^{1 / 2}\right. \\
& \left.+\llbracket \mathfrak{h}_{1}-\mathfrak{h}_{2} \rrbracket^{1 / 2} \llbracket \mathscr{H} \mathfrak{h}_{1}-\mathscr{H} \mathfrak{h}_{2} \rrbracket^{(q-1) / 2}\right) .
\end{aligned}
$$




\section{Hence}

$$
\begin{aligned}
\llbracket \mathscr{H} \mathfrak{h}_{1}-\mathscr{H} \mathfrak{h}_{2} \rrbracket= & \int_{\mathbb{R}^{n}}\left|\mathscr{A} \alpha_{1}-\mathscr{A} \alpha_{2}\right|^{p}+\int_{\mathbb{R}^{n}}\left|\mathscr{B} \beta_{1}-\mathscr{B} \beta_{2}\right|^{q} \\
\preccurlyeq & \llbracket \mathfrak{h}_{1}-\mathfrak{h}_{2} \rrbracket+\llbracket \mathfrak{h}_{1}-\mathfrak{h}_{2} \rrbracket^{q / 2}\left(\llbracket \mathfrak{h}_{1} \rrbracket+\llbracket \mathfrak{h}_{2} \rrbracket\right)^{(2-q) / 2} \\
& +\llbracket \mathfrak{h}_{1}-\mathfrak{h}_{2} \rrbracket^{(q-1) / 2}\left(\llbracket \mathfrak{h}_{1} \rrbracket+\llbracket \mathfrak{h}_{2} \rrbracket\right)^{(2-q) / 2} \llbracket \mathscr{H} \mathfrak{h}_{1}-\mathscr{H} \mathfrak{h}_{2} \rrbracket^{1 / 2} \\
& +\llbracket \mathfrak{h}_{1}-\mathfrak{h}_{2} \rrbracket^{1 / 2}\left(\llbracket \mathfrak{h}_{1} \rrbracket+\llbracket \mathfrak{h}_{2} \rrbracket\right)^{(2-q) / 2} \llbracket \mathscr{H} \mathfrak{h}_{1}-\mathscr{H} \mathfrak{h}_{2} \rrbracket^{(q-1) / 2} .
\end{aligned}
$$

Finally, with the aid of Young's inequalities, we separate $\llbracket \mathscr{H} \mathfrak{h}_{1}-\mathscr{H} \mathfrak{h}_{2} \rrbracket$ from the other terms and then it gets absorbed by the left hand side:

$$
\begin{aligned}
\llbracket \mathscr{H} \mathfrak{h}_{1}-\mathscr{H} \mathfrak{h}_{2} \rrbracket \preccurlyeq & \llbracket \mathfrak{h}_{1}-\mathfrak{h}_{2} \rrbracket+\llbracket \mathfrak{h}_{1}-\mathfrak{h}_{2} \rrbracket^{q / 2}\left(\llbracket \mathfrak{h}_{1} \rrbracket+\llbracket \mathfrak{h}_{2} \rrbracket\right)^{(2-q) / 2} \\
& +\llbracket \mathfrak{h}_{1}-\mathfrak{h}_{2} \rrbracket^{q-1}\left(\llbracket \mathfrak{h}_{1} \rrbracket+\llbracket \mathfrak{h}_{2} \rrbracket\right)^{2-q} \\
& +\llbracket \mathfrak{h}_{1}-\mathfrak{h}_{2} \rrbracket^{1 /(3-q)}\left(\llbracket \mathfrak{h}_{1} \rrbracket+\llbracket \mathfrak{h}_{2} \rrbracket\right)^{(2-q) /(3-q)} \\
\preccurlyeq & \llbracket \mathfrak{h}_{1}-\mathfrak{h}_{2} \rrbracket^{\theta}\left(\llbracket \mathfrak{h}_{1} \rrbracket+\llbracket \mathfrak{h}_{2} \rrbracket\right)^{1-\theta}=\llbracket \mathfrak{h}_{1}-\mathfrak{h}_{2} \rrbracket^{q-1}\left(\llbracket \mathfrak{h}_{1} \rrbracket+\llbracket \mathfrak{h}_{2} \rrbracket\right)^{2-q},
\end{aligned}
$$

where $\theta=\min \{p-1, q-1\}=q-1 \leqslant \min \{q / 2,1 /(3-q)\}$, completing the proof of the Hölder continuity estimate at $(20)$.

3.3. An example. The following example shows that the Hölder exponent $\theta=\min \{p-1, q-1\}=\min \{p / q, q / p\}$ is sharp. Consider the sequence (27) and the trivial one at (28). Given two real functions $\mathfrak{a} \in \mathscr{L}^{p}\left(\mathbb{R}^{n}\right)$ and $\mathfrak{b} \in \mathscr{L}^{q}\left(\mathbb{R}^{n}\right), 1<p \leqslant 2 \leqslant q<\infty$, we can solve the equation

$$
|\mathfrak{a}+\mathscr{A} \alpha|^{p-2}(\mathfrak{a}+\mathscr{A} \alpha)=\mathfrak{b}+\mathscr{B} \beta, \quad \mathscr{B} \equiv 0,
$$

uniquely for $\mathfrak{a}+\mathscr{A} \alpha \in \mathscr{L}^{p}\left(\mathbb{R}^{n}\right)$, where $\mathscr{A}=\operatorname{div}: \mathscr{W}^{1, p}\left(\mathbb{R}^{n}, \mathbb{R}^{n}\right) \rightarrow \mathscr{L}^{p}\left(\mathbb{R}^{n}\right)$. Of course, $\alpha$ is unique only up to a divergence free vector field.

In this way we obtain a $p$-harmonic type operator defined on a pair of dual spaces, $\mathscr{H}: \mathscr{L}^{p}\left(\mathbb{R}^{n}\right) \times \mathscr{L}^{q}\left(\mathbb{R}^{n}\right) \rightarrow \mathscr{L}^{p}\left(\mathbb{R}^{n}\right) \times \mathscr{L}^{q}\left(\mathbb{R}^{n}\right)$. Consider $\mathfrak{h}=$ $(0, \mathfrak{b})$, so that $\mathscr{H} \mathfrak{h}=(\mathscr{A} \alpha, 0)=\left(|\mathfrak{b}|^{q-2} \mathfrak{b}, 0\right)$. In other words, $\mathscr{H}$ restricted to $\{0\} \times \mathscr{L}^{q}\left(\mathbb{R}^{n}\right)$ becomes a nonlinear algebraic transformation of functions. Fix $\mathfrak{b} \in \mathscr{L}^{q}\left(\mathbb{R}^{n}\right)$ whose $q$-norm is one. For a sufficiently small positive number $\varepsilon$ we may consider a perturbation of $\mathfrak{h}$ denoted by $\mathfrak{h}^{\prime}=\mathfrak{h}+\varepsilon \mathfrak{h}$. Thus $\llbracket \mathfrak{h} \rrbracket=\|\mathfrak{b}\|_{q}^{q}=1$, while $\llbracket \mathfrak{h}^{\prime} \rrbracket=(1+\varepsilon)^{q}$ and $\llbracket \mathfrak{h}^{\prime}-\mathfrak{h} \rrbracket=\varepsilon^{q}$. On the other hand $\mathscr{H} \mathfrak{h}^{\prime}-\mathscr{H} \mathfrak{h}=\left[(1+\varepsilon)^{q-1}-1\right]|\mathfrak{b}|^{q-2} \mathfrak{b}$, thus $\llbracket \mathscr{H} \mathfrak{h}^{\prime}-\mathscr{H} \mathfrak{h} \rrbracket=\left[(1+\varepsilon)^{q-1}-1\right]^{p}$. We now see that

$$
\frac{\llbracket \mathscr{H} \mathfrak{h}^{\prime}-\mathscr{H} \mathfrak{h} \rrbracket}{\left[\left[\mathfrak{h}^{\prime}-\mathfrak{h} \rrbracket^{\theta}\left(\llbracket \mathfrak{h}^{\prime} \rrbracket+\llbracket \mathfrak{h} \rrbracket\right)^{1-\theta}\right.\right.}=\frac{\left[(1+\varepsilon)^{q-1}-1\right]^{p}}{\left.\varepsilon^{\theta q}\left[1+(1+\varepsilon)^{q}\right)\right]^{1-\theta}}=O\left(\varepsilon^{p-\theta q}\right) \quad \text { as } \varepsilon \rightarrow 0 .
$$

This ratio stays bounded if and only if $\theta \leqslant p / q=p-1=\min \{p-1, q-1\}$, as claimed. 
4. Interpolation. We follow the idea of Marcinkiewicz [M]. However, the details to be discussed are far from being routine, largely because of the nonlinear terms involved. For the sake of readability we precede the proof with additional notation and some lemmas.

4.1. Notation. Given a pair of functions $\mathfrak{h}=(\mathfrak{a}, \mathfrak{b}) \in \mathscr{L}^{\tau p}\left(\mathbb{R}^{n}, \mathbb{V}\right) \times$ $\mathscr{L}^{\tau q}\left(\mathbb{R}^{n}, \mathbb{V}\right)$, we recall that $[\mathfrak{h}]=|\mathfrak{a}|^{p}+|\mathfrak{b}|^{q} \in \mathscr{L}^{\tau}\left(\mathbb{R}^{n}\right)$. To every positive parameter $t$ there corresponds a decomposition $\mathfrak{h}=\mathfrak{h}^{t}+\mathfrak{h}_{t}$ into truncations of $\mathfrak{h}$, where

$$
\begin{aligned}
& \mathfrak{h}^{t}=\left(\mathfrak{a}^{t}, \mathfrak{b}^{t}\right)= \begin{cases}\mathfrak{h}(x) & \text { if }[\mathfrak{h}(x)] \geqslant t, \\
0 & \text { if }[\mathfrak{h}(x)]<t,\end{cases} \\
& \mathfrak{h}_{t}=\left(\mathfrak{a}_{t}, \mathfrak{b}_{t}\right)= \begin{cases}0 & \text { if }[\mathfrak{h}(x)] \geqslant t, \\
\mathfrak{h}(x) & \text { if }[\mathfrak{h}(x)]<t\end{cases}
\end{aligned}
$$

Next, we recall the operator $\mathscr{H}$ and the associated equations

$$
\mathscr{H} \mathfrak{h}=(\mathscr{A} \alpha, \mathscr{B} \beta), \quad \mathbf{A}(x, \mathfrak{a}+\mathscr{A} \alpha)=\mathfrak{b}+\mathscr{B} \beta .
$$

In the spirit of Section 3.2, we abbreviate the notation by setting $\mathcal{A}=$ $\mathfrak{a}+\mathscr{A} \alpha, \mathcal{B}=\mathfrak{b}+\mathscr{B} \beta$ and $\mathcal{H}=(\mathcal{A}, \mathcal{B})$. Furthermore, we write

$$
[\mathcal{H}]=|\mathcal{A}|^{p}+|\mathcal{B}|^{q}, \quad \mathbf{A}(x, \mathcal{A})=\mathcal{B} .
$$

Analogous equations with subscript and superscript $t$ will also come into play:

$$
\begin{aligned}
\mathscr{H} \mathfrak{h}_{t} & =\left(\mathscr{A} \alpha_{t}, \mathscr{B} \beta_{t}\right), & \mathbf{A}\left(x, \mathfrak{a}_{t}+\mathscr{A} \alpha_{t}\right) & =\mathfrak{b}_{t}+\mathscr{B} \beta_{t}, \\
{\left[\mathcal{H}_{t}\right] } & =\left|\mathcal{A}_{t}\right|^{p}+\left|\mathcal{B}_{t}\right|^{q}, & \mathbf{A}\left(x, \mathcal{A}_{t}\right) & =\mathcal{B}_{t}, \\
\mathscr{H} \mathfrak{h}^{t} & =\left(\mathscr{A} \alpha^{t}, \mathscr{B} \beta^{t}\right), & \mathbf{A}\left(x, \mathfrak{a}^{t}+\mathscr{A} \alpha^{t}\right) & =\mathfrak{b}^{t}+\mathscr{B} \beta^{t}, \\
{\left[\mathcal{H}^{t}\right] } & =\left|\mathcal{A}^{t}\right|^{p}+\left|\mathcal{B}^{t}\right|^{q}, & \mathbf{A}\left(x, \mathcal{A}^{t}\right) & =\mathcal{B}^{t} .
\end{aligned}
$$

Without loss of generality we may assume that $1<q \leqslant 2 \leqslant p$. Then there are two cases to consider: $1<\tau<\lambda$ and $\lambda<\tau<1$. The case $\tau=1$, corresponding to the natural domain of definition, has already been established in Section 3.1. We need only consider the first case. The reader may wish to carefully inspect our arguments to produce the proof of the second case by simply permuting subscripts and superscripts. Let us point out that the equations (48) will play no role in the first case; they are important for the second case.

4.2. Three lemmas. In this subsection we collect some elementary algebraic facts.

LEMmA 4.1. Under the assumption $1<q \leqslant 2 \leqslant p$, we have

$$
\begin{aligned}
\left|\mathcal{A}-\mathcal{A}_{t}\right|^{p} & \preccurlyeq\left\langle\mathcal{A}-\mathcal{A}_{t} \mid \mathcal{B}-\mathcal{B}_{t}\right\rangle, \\
\left|\mathcal{B}-\mathcal{B}_{t}\right|^{q} & \preccurlyeq|\mathcal{B}|^{q}+\left\langle\mathcal{A}-\mathcal{A}_{t} \mid \mathcal{B}-\mathcal{B}_{t}\right\rangle, \\
\left|\mathcal{B}-\mathcal{B}_{t}\right|^{q} & \preccurlyeq\left|\mathcal{B}_{t}\right|^{q}+\left\langle\mathcal{A}-\mathcal{A}_{t} \mid \mathcal{B}-\mathcal{B}_{t}\right\rangle .
\end{aligned}
$$


Proof. It follows from (45) and (47) that

$$
\begin{aligned}
\left\langle\mathbf{A}(x, \mathcal{A})-\mathbf{A}\left(x, \mathcal{A}_{t}\right) \mid \mathcal{A}-\mathcal{A}_{t}\right\rangle & =\left\langle\mathcal{A}-\mathcal{A}_{t} \mid \mathcal{B}-\mathcal{B}_{t}\right\rangle \\
& =\left\langle\mathbf{B}(x, \mathcal{B})-\mathbf{B}\left(x, \mathcal{B}_{t}\right) \mid \mathcal{B}-\mathcal{B}_{t}\right\rangle .
\end{aligned}
$$

On account of conditions (14b) and (16c), we have the following chain of inequalities:

$$
\begin{aligned}
\left|\mathcal{A}-\mathcal{A}_{t}\right|^{p} & \leqslant\left|\mathcal{A}-\mathcal{A}_{t}\right|^{2}\left(|\mathcal{A}|+\left|\mathcal{A}_{t}\right|\right)^{p-2} \\
& \preccurlyeq \succcurlyeq\left\langle\mathcal{A}-\mathcal{A}_{t} \mid \mathcal{B}-\mathcal{B}_{t}\right\rangle \\
& \preccurlyeq \succcurlyeq\left|\mathcal{B}-\mathcal{B}_{t}\right|^{2}\left(|\mathcal{B}|+\left|\mathcal{B}_{t}\right|\right)^{q-2} \leqslant\left|\mathcal{B}-\mathcal{B}_{t}\right|^{q} .
\end{aligned}
$$

Observe that the inequality (49) appears in the middle of this chain. Concerning (50) and (51), the reader may wish to check the following general inequality, which holds in any normed space:

$$
\left|\mathcal{B}-\mathcal{B}_{t}\right|^{q} \preccurlyeq\left|\mathcal{B}_{t}\right|^{q}+\left|\mathcal{B}-\mathcal{B}_{t}\right|^{2}\left(|\mathcal{B}|+\left|\mathcal{B}_{t}\right|\right)^{q-2} .
$$

Permuting $\mathcal{B}$ and $\mathcal{B}_{t}$ gives us another one:

$$
\left|\mathcal{B}-\mathcal{B}_{t}\right|^{q} \preccurlyeq|\mathcal{B}|^{q}+\left|\mathcal{B}-\mathcal{B}_{t}\right|^{2}\left(|\mathcal{B}|+\left|\mathcal{B}_{t}\right|\right)^{q-2} .
$$

The proof of Lemma 4.1 is complete.

In the next step we integrate those inequalities:

LEMma 4.2. The notation being as above, we have

$$
\int_{\mathbb{R}^{n}}\left\langle\mathcal{A}-\mathcal{A}_{t} \mid \mathcal{B}-\mathcal{B}_{t}\right\rangle \preccurlyeq \llbracket \mathfrak{h}^{t} \rrbracket+\int_{\mathbb{R}^{n}}\left[\mathfrak{h}^{t}\right]^{1 / p}[\mathcal{H}]^{1 / q} .
$$

The point of this estimate is that both terms $\left[\mathfrak{h}^{t}\right]^{1 / p}$ and $[\mathcal{H}]^{1 / q}$ appear under the same integral sign. Our subsequent arguments would not work if at this point we had used Hölder's inequality to separate the two terms.

Proof. Recall the notation: $\mathcal{A}=\mathfrak{a}+\mathscr{A} \alpha, \mathcal{B}=\mathfrak{b}+\mathscr{B} \beta, \mathcal{A}_{t}=\mathfrak{a}_{t}+\mathscr{A} \alpha_{t}$ and $\mathcal{B}_{t}=\mathfrak{b}_{t}+\mathscr{B} \beta_{t}$. Moreover $\mathfrak{a}^{t}=\mathfrak{a}-\mathfrak{a}_{t}$ and $\mathfrak{b}^{t}=\mathfrak{b}-\mathfrak{b}_{t}$. We express the integral on the left hand side as

$$
\begin{aligned}
\int_{\mathbb{R}^{n}}\left\langle\mathcal{A}-\mathcal{A}_{t} \mid \mathcal{B}-\mathcal{B}_{t}\right\rangle= & \int_{\mathbb{R}^{n}}\left\langle\mathfrak{a}^{t} \mid \mathcal{B}-\mathcal{B}_{t}\right\rangle+\int_{\mathbb{R}^{n}}\left\langle\mathcal{A}-\mathcal{A}_{t} \mid \mathfrak{b}^{t}\right\rangle \\
& -\int_{\mathbb{R}^{n}}\left\langle\mathfrak{a}^{t} \mid \mathfrak{b}^{t}\right\rangle+\int_{\mathbb{R}^{n}}\left\langle\mathscr{A}\left(\alpha-\alpha_{t}\right) \mid \mathscr{B}\left(\beta-\beta_{t}\right)\right\rangle .
\end{aligned}
$$

The last integral vanishes because $\operatorname{Im} \mathscr{A}$ and $\operatorname{Im} \mathscr{B}$ are orthogonal (see Lemma 2.1). Now the calculation continues as follows:

$$
\begin{aligned}
& \int_{\mathbb{R}^{n}}\left\langle\mathcal{A}-\mathcal{A}_{t} \mid \mathcal{B}-\mathcal{B}_{t}\right\rangle \leqslant \llbracket \mathfrak{h}^{t} \rrbracket+\int_{\mathbb{R}^{n}}\left|\mathfrak{a}^{t}\right|\left|\mathcal{B}-\mathcal{B}_{t}\right|+\int_{\mathbb{R}^{n}}\left|\mathcal{A}-\mathcal{A}_{t}\right|\left|\mathfrak{b}^{t}\right| \\
& \leqslant \llbracket \mathfrak{h}^{t} \rrbracket+\int_{\mathbb{R}^{n}}\left[\mathfrak{h}^{t}\right]^{1 / p}|\mathcal{B}|+\int_{\mathbb{R}^{n}}\left[\mathfrak{h}^{t}\right]^{1 / p}\left\langle\mathcal{A}-\mathcal{A}_{t} \mid \mathcal{B}-\mathcal{B}_{t}\right\rangle^{1 / q}+\int_{\mathbb{R}^{n}}\left[\mathfrak{h}^{t}\right]^{1 / q}\left\langle\mathcal{A}-\mathcal{A}_{t} \mid \mathcal{B}-\mathcal{B}_{t}\right\rangle^{1 / p}
\end{aligned}
$$


by (50) and (49), respectively. With the aid of Young's inequality we can separate the inner product $\left\langle\mathcal{A}-\mathcal{A}_{t} \mid \mathcal{B}-\mathcal{B}_{t}\right\rangle$ from $\left[\mathfrak{h}^{t}\right]$ and get it absorbed by the left hand side of (56). We then conclude with the estimate

$$
\int_{\mathbb{R}^{n}}\left\langle\mathcal{A}-\mathcal{A}_{t} \mid \mathcal{B}-\mathcal{B}_{t}\right\rangle \preccurlyeq \llbracket \mathfrak{h}^{t} \rrbracket+\int_{\mathbb{R}^{n}}\left[\mathfrak{h}^{t}\right]^{1 / p}|B| \preccurlyeq \llbracket \mathfrak{h}^{t} \rrbracket+\int_{\mathbb{R}^{n}}\left[\mathfrak{h}^{t}\right]^{1 / p}|\mathcal{H}|^{1 / q}
$$

as claimed.

Lemma 4.3. For every $1<\tau<\lambda$, we have

$$
\int_{0}^{\infty} t^{\tau-1} \operatorname{meas}\left\{x ;\left[\mathcal{H}_{t}(x)\right]>t\right\} \mathrm{d} t \preccurlyeq \llbracket \mathfrak{h} \rrbracket_{\tau}^{\tau} .
$$

Proof. By the notation at $(47)$ we see that $\left[\mathcal{H}_{t}\right]=\left[\mathfrak{h}_{t}+\mathscr{H} \mathfrak{h}_{t}\right] \preccurlyeq\left[\mathfrak{h}_{t}\right]+$ $\left[\mathscr{H} \mathfrak{h}_{t}\right]$. Hence the line integral in (57) splits as

$$
\int_{0}^{\infty} t^{\tau-1} \operatorname{meas}\left\{x ;\left[\mathfrak{h}_{t}(x)\right]>t\right\} \mathrm{d} t+\int_{0}^{\infty} t^{\tau-1} \operatorname{meas}\left\{x ;\left[\mathscr{H} \mathfrak{h}_{t}(x)\right]>t\right\} \mathrm{d} t .
$$

Since both $\mathscr{H}$ and the identity operator are of weak type $\lambda$, we can estimate each of these terms by the same double integral:

$$
\int_{0}^{\infty} t^{\tau-1}\left(t^{-\lambda} \int_{\mathbb{R}^{n}}\left[\mathfrak{h}_{t}(x)\right]^{\lambda} \mathrm{d} x\right) \mathrm{d} t=\int_{0}^{\infty} t^{\tau-\lambda-1}\left(\int_{[\mathfrak{h}]>t}[\mathfrak{h}(x)]^{\lambda} \mathrm{d} x\right) \mathrm{d} t .
$$

Applying Fubini's theorem yields

$$
\begin{aligned}
\int_{0}^{\infty} t^{\tau-1} \operatorname{meas}\left\{x ;\left[\mathcal{H}_{t}(x)\right]>t\right\} \mathrm{d} t & \preccurlyeq \int_{\mathbb{R}^{n}}[\mathfrak{h}(x)]^{\lambda}\left(\int_{[\mathfrak{h}]}^{\infty} t^{\tau-\lambda-1} \mathrm{~d} t\right) \mathrm{d} x \\
& \preccurlyeq \int_{\mathbb{R}^{n}}[\mathfrak{h}(x)]^{\tau} \mathrm{d} x=\llbracket \mathfrak{h} \rrbracket_{\tau}^{\tau},
\end{aligned}
$$

completing the proof of Lemma 4.3 .

With these three lemmas at hand we now proceed to the final step of the interpolation.

4.3. Proof of the Interpolation Theorem. The aim is to estimate the integrals

$$
\int_{\mathbb{R}^{n}}|\mathscr{H} \mathfrak{h}|^{\tau} \preccurlyeq \int_{\mathbb{R}^{n}}|h|^{\tau}+\int_{\mathbb{R}^{n}}[\mathcal{H}(x)]^{\tau} \mathrm{d} x
$$

in terms of $\llbracket \mathfrak{h} \rrbracket_{\tau}^{\tau}$. We shall draw on a somewhat similar computation from E. Stein $[S]$ :

$$
\begin{aligned}
& \int_{\mathbb{R}^{n}}[\mathcal{H}(x)]^{\tau} \mathrm{d} x=\tau \int_{0}^{\infty} t^{\tau-1} \operatorname{meas}\{x ;[\mathcal{H}(x)]>t\} \mathrm{d} t \\
& \quad \preccurlyeq \int_{0}^{\infty} t^{\tau-1} \operatorname{meas}\left\{x ;\left[\mathcal{H}_{t}(x)\right]>t\right\} \mathrm{d} t+\int_{0}^{\infty} t^{\tau-1} \operatorname{meas}\left\{x ;\left[\mathcal{H}(x)-\mathcal{H}_{t}(x)\right]>t\right\} \mathrm{d} t .
\end{aligned}
$$


In view of Lemma 4.3 the first integral is bounded by $\llbracket \mathfrak{h} \rrbracket_{\tau}^{\tau}$. To estimate the second integral we observe, using Lemma 4.1, that

$$
\begin{aligned}
{\left[\mathcal{H}-\mathcal{H}_{t}\right] } & =\left|\mathcal{A}-\mathcal{A}_{t}\right|^{p}+\left|\mathcal{B}-\mathcal{B}_{t}\right|^{q} \preccurlyeq\left|\mathcal{B}_{t}\right|^{q}+\left\langle\mathcal{A}-\mathcal{A}_{t} \mid \mathcal{B}-\mathcal{B}_{t}\right\rangle \\
& \preccurlyeq\left[\mathcal{H}_{t}\right]+\left\langle\mathcal{A}-\mathcal{A}_{t} \mid \mathcal{B}-\mathcal{B}_{t}\right\rangle .
\end{aligned}
$$

Accordingly, the second integral splits as

$$
\begin{aligned}
& \int_{0}^{\infty} t^{\tau-1} \operatorname{meas}\{x ;[\mathcal{H}(x)-\left.\left.\mathcal{H}_{t}(x)\right]>t\right\} \mathrm{d} t \\
& \preccurlyeq \int_{0}^{\infty} t^{\tau-1} \operatorname{meas}\left\{x ;\left[\mathcal{H}_{t}(x)\right]>t\right\} \mathrm{d} t \\
&+\int_{0}^{\infty} t^{\tau-1} \operatorname{meas}\left\{x ;\left\langle\mathcal{A}-\mathcal{A}_{t} \mid \mathcal{B}-\mathcal{B}_{t}\right\rangle>t\right\} \mathrm{d} t \\
& \preccurlyeq \llbracket \mathfrak{h} \rrbracket_{\tau}^{\tau}+\int_{0}^{\infty} t^{\tau-2}\left(\int_{\mathbb{R}^{n}}\left\langle\mathcal{A}-\mathcal{A}_{t} \mid \mathcal{B}-\mathcal{B}_{t}\right\rangle \mathrm{d} x\right) \mathrm{d} t
\end{aligned}
$$

again by Lemma 4.3. Using Lemma 4.2 we continue this chain of inequalities:

$$
\preccurlyeq \llbracket \mathfrak{h} \rrbracket_{\tau}^{\tau}+\int_{0}^{\infty} t^{\tau-2}\left(\int_{[\mathfrak{h}] \geqslant t}[\mathfrak{h}]^{1 / p}[\mathcal{H}]^{1 / q}\right) \mathrm{d} t .
$$

It is worthwhile to emphasize again that both terms $[\mathfrak{h}]^{1 / p}$ and $[\mathcal{H}]^{1 / q}$ appear under one integral sign. Applying Fubini's theorem yields

$$
\begin{aligned}
& =\llbracket \mathfrak{h} \rrbracket_{\tau}^{\tau}+\int_{\mathbb{R}^{n}}[\mathfrak{h}]^{1 / p}[\mathcal{H}]^{1 / q} \int_{0}^{[\mathfrak{h}]} t^{\tau-2} \mathrm{~d} t \\
& =\llbracket \mathfrak{h} \rrbracket_{\tau}^{\tau}+\frac{1}{\tau-1} \int_{\mathbb{R}^{n}}[\mathfrak{h}]^{\tau-1 / q}[\mathcal{H}]^{1 / q} .
\end{aligned}
$$

Now the integral we are dealing with can be estimated as follows:

$$
\int_{\mathbb{R}^{n}}[\mathcal{H}]^{\tau} \preccurlyeq \llbracket \mathfrak{h} \rrbracket_{\tau}^{\tau}+\int_{\mathbb{R}^{n}}[\mathfrak{h}]^{\tau-1 / q}[\mathcal{H}]^{1 / q} .
$$

It is at this point that we shall finally separate $[\mathcal{H}]$ from $[\mathfrak{h}]$. As usual, the device we may use here is Young's inequality:

$$
\int_{\mathbb{R}^{n}}[\mathcal{H}]^{\tau} \preccurlyeq \llbracket \mathfrak{h} \rrbracket_{\tau}^{\tau}+C_{\varepsilon} \int_{\mathbb{R}^{n}}[\mathfrak{h}]^{\tau}+\varepsilon \int_{\mathbb{R}^{n}}[\mathcal{H}]^{\tau} .
$$

The last term will be absorbed by the left hand side once we choose $\varepsilon$ sufficiently small. This results in the estimate

$$
\int_{\mathbb{R}^{n}}[\mathcal{H}]^{\tau} \preccurlyeq \llbracket \mathfrak{h} \rrbracket_{\tau}^{\tau}
$$

The proof is completed by noticing that $\mathscr{H} \mathfrak{h}=\mathcal{H}-\mathfrak{h}$. 
REMARK. It is certainly curious that in our computation the expected uniform bounds are lost when $\tau$ approaches 1 , because of the inequality at (59). This situation also occurs for linear operators, if we know only weak type estimates at the end-points of the interpolation. In our case, however, we know that $\mathscr{H}$ is of strong type 1 . It may very well be possible to end up with uniform estimates as $\tau \rightarrow 1$ by some more refined arguments.

\section{References}

[B] L. Bers, Mathematical Aspects of Subsonic and Transonic Gas Dynamics, Surveys Appl. Math. 3, Wiley, New York, and Chapman and Hall, London, 1958.

[BI.1] B. Bojarski and T. Iwaniec, Analytical foundations of the theory of quasiconformal mappings in $R^{n}$, Ann. Acad. Sci. Fenn. Ser. A I Math. 8 (1983), $257-324$.

[BI.2] - - - p-harmonic equation and quasiregular mappings, in: Partial Differential Equations (Warszawa, 1984), Banach Center Publ. 19, PWN, Warszawa, 1987, 25-38; see also Universität Bonn, Sonderforschungsbereich 72, Preprint 617, 1-20 (1983).

[Br] F. E. Browder, Nonlinear elliptic boundary value problems, Bull. Amer. Math. Soc. 69 (1963), 862-874.

[CCRSW] R. R. Coifman, M. Cwikel, R. Rochberg, Y. Sagher and G. Weiss, A theory of complex interpolation for families of Banach spaces, Adv. Math. 43 (1982), 203-229.

[DM] E. DiBenedetto and J. Manfredi, On the higher integrability of the gradient of weak solutions of certain degenerate elliptic systems, Amer. J. Math. 115 (1993), 1107-1134.

[DI] L. D'Onofrio and T. Iwaniec, The p-harmonic transform beyond its natural domain of definition, Indiana Univ. Math. J., to appear.

[GV] F. Giannetti and A. Verde, Variational integrals for elliptic complexes, Studia Math. 140 (2000), 79-98.

[GIS] L. Greco, T. Iwaniec and C. Sbordone, Inverting the p-harmonic operator, Manuscripta Math. 92 (1997), 249-258.

[HKM] J. Heinonen, T. Kilpeläinen and O. Martio, Nonlinear Potential Theory of Degenerate Elliptic Equations, Oxford Math. Monogr., Oxford Univ. Press, New York, 1993.

[I.1] T. Iwaniec, On $L^{p}$-integrability in PDEs and quasiregular mappings for large exponents, Ann. Acad. Sci. Fenn. Ser. A I Math. 7 (1982), 301-322.

[I.2] - Projections onto gradient fields and $L^{p}$-estimates for degenerated elliptic operators, Studia Math. 75 (1983), 293-312.

[I.3] - $\quad$-harmonic tensors and quasiregular mappings, Ann. of Math. (2) 136 (1992), 589-624.

[I.4] -, Nonlinear commutators and Jacobians, J. Fourier Anal. Appl. 3 (1997), 775-796.

[IMa] T. Iwaniec and J. Manfredi, Regularity of p-harmonic functions on the plane, Rev. Mat. Iberoamericana 5 (1989), 1-19.

[IM] T. Iwaniec and G. Martin, Geometric Function Theory and Non-linear Analysis, Oxford Math. Monogr., Oxford Univ. Press, New York, 2001. 
[IS.1] T. Iwaniec and C. Sbordone, Weak minima of variational integrals, J. Reine Angew. Math. 454 (1994), 143-161.

[IS.2] - - - Quasiharmonic fields, Ann. Inst. H. Poincaré Anal. Non Linéaire 18 (2001), 519-572.

[ISS] T. Iwaniec, C. Scott and B. Stroffolini, Nonlinear Hodge theory on manifolds with boundary, Ann. Mat. Pura Appl. (4) 177 (1999), 37-115.

[JLM] P. Juutinen, P. Lindqvist and J. Manfredi, The infinity Laplacian: examples and observations, Papers on analysis, 207-217, Report. Univ. of Jyväskylä, Dept. Math. Statist. 83, Jyväskylä, 2001.

[MaZ] J. Malý and W. P. Ziemer, Fine Regularity of Solutions of Elliptic Partial Differential Equations, Math. Surveys Monogr. 51, Amer. Math. Soc., 1997.

[M] J. Marcinkiewicz, Sur l'interpolation d'opérations, C. R. Acad. Sci. Paris 208 (1939), 1272-1273.

[Mi] G. J. Minty, Monotone (nonlinear) operators in Hilbert space, Duke Math. J. 29 (1962), 341-346.

[Re] Y. G. Reshetnyak, Space Mappings with Bounded Distortion, translated from the Russian by H. H. McFaden, Transl. Math. Monogr. 73, Amer. Math. Soc., Providence, RI, 1989.

[Ri] S. Rickman, Quasiregular Mappings, Ergeb. Math. Grenzgeb. 26, Springer, Berlin, 1993.

[RW] R. Rochberg and G. Weiss, Derivatives of analytic families of Banach spaces, Ann. of Math. (2) 118 (1983), 315-347.

[S] E. M. Stein, Singular Integrals and Differentiability Properties of Functions, Princeton Math. Ser. 30, Princeton Univ. Press, Princeton, NJ, 1970.

[Uh] K. Uhlenbeck, Regularity for a class of non-linear elliptic systems, Acta Math. 138 (1977), 219-240.

[U] N. N. Ural'tseva, Degenerate quasilinear elliptic systems, Zap. Nauchn. Sem. Leningrad. Otdel. Mat. Inst. Steklov. (LOMI) 7 (1968), 184-222 (in Russian).

Dipartimento di Matematica

e Applicazioni "R. Caccioppoli"

Complesso Universitario Monte S. Angelo

80126, Napoli, Italy

E-mail: ldonofri@unina.it
Department of Mathematics

Syracuse University

Syracuse, NY 13244, U.S.A. E-mail: tiwaniec@mailbox.syr.edu

Received December 11, 2002

Revised version April 11, 2003 